\title{
Structural determinants of human $\zeta$-globin mRNA stability
}

\author{
Zhenning $\mathrm{He}^{1}$, Decheng Song ${ }^{1}$, Sebastiaan van Zalen ${ }^{1}$ and J Eric Russell ${ }^{1,2^{*}}$
}

\begin{abstract}
Background: The normal accumulation of adult $\alpha$ and $\beta$ globins in definitive erythrocytes is critically dependent upon processes that ensure that the cognate mRNAs are maintained at high levels in transcriptionally silent, but translationally active progenitor cells. The impact of these post-transcriptional regulatory events on the expression of embryonic $\zeta$ globin is not known, as its encoding mRNA is not normally transcribed during adult erythropoiesis. Recently, though, $\zeta$ globin has been recognized as a potential therapeutic for a thalassemia and sickle-cell disease, raising practical questions about constitutive post-transcriptional processes that may enhance, or possibly prohibit, the expression of exogenous or derepresssed endogenous $\zeta$-globin genes in definitive erythroid progenitors.
\end{abstract}

Methods: The present study assesses mRNA half-life in intact cells using a pulse-chase approach; identifies cis-acting determinants of $\zeta$-globin mRNA stability using a saturation mutagenesis strategy; establishes critical 3'UTR secondary structures using an in vitro enzymatic mapping method; and identifies trans-acting effector factors using an affinity chromatographical procedure.

Results: We specify a tetranucleotide $3^{\prime} U T R$ motif that is required for the high-level accumulation of $\zeta-g l o b i n$ transcripts in cultured cells, and formally demonstrate that it prolongs their cytoplasmic half-lives. Surprisingly, the $\zeta$-globin mRNA stability motif does not function autonomously, predicting an activity that is subject to structural constraints that we subsequently specify. Additional studies demonstrate that the $\zeta$-globin mRNA stability motif is targeted by AUF1, a ubiquitous RNA-binding protein that enhances the half-life of adult $\beta$-globin mRNA, suggesting commonalities in post-transcriptional processes that regulate globin transcripts at all stages of mammalian development.

Conclusions: These data demonstrate a mechanism for Z-globin mRNA stability that exists in definitive erythropoiesis and is available for therapeutic manipulation in a thalassemia and sickle-cell disease.

Keywords: RNA stability, $\zeta$ Globin, Sickle-cell disease, Thalassemia

\section{Background}

Human $\alpha$-like globins are encoded by three homologous genes $\left(5^{\prime}-\zeta-\alpha 2-\alpha 1-3^{\prime}\right)$ arranged in order of their developmental expression [1]. Embryonic $\alpha$-like $\zeta$ globin is produced during the first gestational trimester in primitive erythroblasts that originate in blood islands of the yolk sac, while fetal/adult $\alpha$ globin is induced at the end of this interval and continues to express in definitive erythroblasts that are initially produced in the liver and, subsequently, in the bone marrow $[1,2]$. Unlike $\alpha$ globin-which is required

\footnotetext{
* Correspondence: jeruss@mail.med.upenn.edu

${ }^{1}$ Department of Medicine (Hematology/Oncology), Perelman School of Medicine at the University of Pennsylvania, Biomedical Research Building, Room 808, 421 Curie Boulevard, Philadelphia, PA 19104, USA

${ }^{2}$ Department of Pediatrics (Hematology), Perelman School of Medicine at the University of Pennsylvania, Philadelphia, PA, USA
}

for normal growth and development both in the uterus and after birth $-\zeta$ globin appears to be largely dispensable to mammalian reproduction. Embryonic $\zeta$ globin is not required for viability in mice [3], and naturally occurring deletions and duplications in its encoding gene are not associated with any defined phenotype in man $[2,4,5]$. As a consequence of this apparent biological irrelevance, then, there has been little incentive to detail the processes that control $\zeta$-globin expression in either primitive or definitive erythropoiesis.

Investigations of the molecular and cellular programs that regulate $\zeta$ globin, though, are recently justified by its demonstrated potential as a novel therapeutic for both $\alpha$ thalassemia and sickle-cell disease [6,7]. When compelled to express in definitive erythroid cells, embryonic $\zeta$ globin 
assembles with adult $\beta$ globin into heterotetrameric $\mathrm{Hb}$ $\zeta_{2} \beta_{2}$ (Hb Portland-2), which exhibits $\mathrm{O}_{2}$-binding and allosteric properties that differ modestly from those of $\mathrm{Hb}$ $\alpha_{2} \beta_{2}$ (Hb A, the principal adult hemoglobin), but that remain fully compatible with normal adult physiology $[8,9]$. The significance of this approach for treating $\alpha-$ globin deficiency states ( $\alpha$ thalassemia) is illustrated by evidence that transgenic human $\zeta$ globin fully reverts the pathological phenotype of mice containing heterozygous knockout of their endogenous $\alpha$-globin genes and, remarkably, restores full viability to animals with homozygous, embryonic-lethal inactivation of these same genes [6]. The developmental stage-discordant expression of $\zeta$ globin holds additional therapeutic promise for sickle-cell disease, as its low-level expression effectively mitigates the abnormal phenotype of mouse models of this disorder $[7,10]$. Importantly, the $\alpha \rightarrow \zeta$ exchange that converts pathological $\mathrm{Hb} \alpha_{2} \beta_{2}^{\mathrm{S}}$ (Hb S) to non-pathological $\mathrm{Hb} \zeta_{2} \beta_{2}^{\mathrm{S}}$ does not exclude the mutant $\beta^{S}$ subunit, raising the possibility that this novel strategy can be coordinated with contemporary therapies that promote $\beta^{S} \rightarrow \gamma$ exchange. These two developing applications for $\zeta$ globin-effected through either gene-reactivation or gene-supplementation strategiesnow recommend careful description of the events that contribute to its regulation in definitive erythroid cells.

Post-transcriptional regulatory processes are critical to the normal accumulation of adult $\alpha$ - and $\beta$-globins in definitive erythrocytes, ensuring that the cognate mRNAs are maintained at high levels in transcriptionally silent-but translationally active-progenitor cells. The fundamental bases for the prolonged half-life $\left(t_{1 / 2}\right)$ values for both $\alpha$ and $\beta$-globin mRNAs have now been established in detail, including the identification of specific trans-acting effector proteins and the cis-acting regulatory elements that they target [11-16]. Similar analyses of embryonic $\zeta$-globin transcripts that are expressed either physiologically or following therapeutic derepression in definitive erythropoiesis indicate that the mRNAs are subject to posttranscriptional processes that are constitutive to these cells $[17,18]$. While $\zeta$-globin mRNA is observed at low levels in normal adult erythroid progenitors [19-21], it can accumulate to significantly higher levels in cells where its encoding gene is transcriptionally dysregulated [22-24], implicating the presence of active post-transcriptional processes that are capable of acting upon developmental stage-discordant $\zeta$-globin mRNA. Transgenic human $\zeta$ globin can also be produced in abundance in adult mice (at levels that support viability in $\alpha$-globin nullizygotes) [6], validating the assertion that-as a practical matter-the half-life of its encoding mRNA is sufficiently long to ensure that biologically relevant levels of $\zeta$ globin are translated [6-9]. These principles are confirmed in individuals with Southeast Asian-type $\alpha$ thalassemia [25], congenital dyserythropoietic anemia [26], and juvenile chronic myelocytic leukemia
[24], who express measurable quantities of both $\zeta$-globin mRNA and $\zeta$-globin protein. Collectively, these observations endorse the likelihood that post-transcriptional programs in adult erythropoiesis are fully permissive for the expression of $\zeta$-globin mRNA.

The present work describes structures within the $\zeta$ globin 3'UTR that are critical to its post-transcriptional regulation, illustrates their effects on the half-life value of the full-length $\zeta$-globin mRNA in intact cells, and identifies a trans-acting factor that is likely to mediate this process. These results provide a foundation for understanding the impact of post-transcriptional activities to the developmental stage-discordant expression of $\zeta$ globin in adult erythropoiesis, as a promising therapeutic for both $\alpha$ thalassemia and sickle-cell disease.

\section{Results}

A transcriptional chase strategy identifies cis-acting regulatory determinants within the $\zeta$-globin $3^{\prime} U T R$

Transgenic human $\zeta$ globin accumulates to high levels in mouse erythrocytes, consistent with the existence of cisacting determinants that stabilize the cognate $\zeta$-globin mRNA in transcriptionally silent progenitor cells $[6,7,18]$. Precedent analyses of both globin and non-globin mRNAs indicate that relevant stability determinants are commonly confined to the 3' region of untranslated mRNA (3'UTR), which is not subject to disruption by translating ribosomes [27-31]. We investigated the likely positioning of an mRNA stability element in the $\zeta$-globin $3^{\prime}$ UTR in a systematic manner, employing a linker-scanning strategy to identify cis-acting determinants of mRNA half-life that act in vivo in intact cells. Full-length $\zeta$-globin genes were constructed to include either the 105-nt wild-type $\zeta$-globin 3'UTR

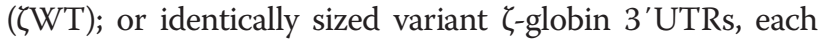
containing an 8-nt mutation at a different position between the UGA translational termination codon and the polyadenylate tail (Figure 1). Each gene was linked to a recombinant hybrid tetracycline response element (TRE) that promotes transcription in cultured cells expressing a hybrid tetracycline trans-activating protein (tTA), but is transcriptionally silent in the presence of tetracycline (tet) [32]. This approach permitted half-life values for wild-type and 3'UTR-variant $\zeta$-globin mRNAs to be specified in vivo in tTA-expressing cells, using a transcriptional chase strategy that assesses the temporal reduction in the level of each variant $\zeta$-globin mRNA relative to the steady-state level of a tet-indifferent control mRNA [33,34].

To identify the positions of mRNA stability determinants within the $\zeta$-globin 3'UTR, we conducted a screening assay that quantified the relative decay of wild-type and 3'UTRvariant $\zeta$-globin mRNAs in vivo in intact cultures. Mammalian cultured-cell models for erythroid development transcribe a variety of embryonic, fetal, and adult globin mRNAs that can compete for post-transcriptional 
regulatory activities $[18,35,36]$; consequently, we conducted our studies in tTA-expressing HeLa cells $\left(\mathrm{HeLa}^{\mathrm{tTA}}\right)$ that do not transcribe globin mRNAs and have previously been used to characterize post-transcriptional processes affecting both native and exogenous globin genes [16,34]. We tested the relative decays of transiently expressed ' $/ \mathrm{WT}$ and 3' UTR-variant $\zeta$-globin mRNAs, relative to that of a control $\beta$-globin mRNA transcribed from a co-transfected gene, using a two-point decay method of our design. One of the 3'UTR-variant mRNAs-containing a CCCCAGCC $\rightarrow$ agtgcaCa substitution at nts 57-64 ( 57 mRNA)-reproducibly decayed over a 16-hour interval to a level that was approximately one-third that of $\zeta W T$ mRNA (Figure 2A). The effect of the octanucleotide substitution was dependent upon its position rather than its content, as $\zeta$-globin mRNAs containing a similar mutation elsewhere in the $3^{\prime} \mathrm{UTR}$-including flanking nts 49-56 and 65-72 ( $\zeta 49$ and $\zeta 65$ mRNAs, respectively)-decayed at the same rate as parental (WWT mRNA. The mutational effects were consistent over three or more replicate analyses, thus identifying and localizing a previously unknown post-transcriptional determinant of mRNA decay within the $\zeta$-globin 3'UTR. For convenience, we now term the nt 57-64 region the $\zeta$-globin mRNA regulatory element (ZMRE).

We posited that the kinetics of $\zeta$-globin mRNA decay might be similarly affected by a smaller defect in the nt 57-64 target region, according with precedent studies demonstrating the deleterious effects of site-specific single- or several-nt 3'UTR substitutions on the stabilities of both $\alpha$ - and $\beta$-globin mRNAs $[16,18,38]$. This hypothesis was validated by transcriptional chase analyses of transiently expressed $\zeta$-globin mRNAs carrying tetranucleotide substitutions at either positions $1-4$ or 5-8 of the

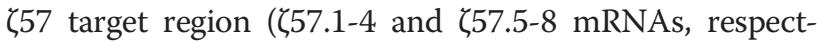
ively; CCCCAGCC $\rightarrow$ agtgAGCC and CCCCAGCC $\rightarrow$ CCCCcaCa). While the relative decay of $\zeta 57.5-8 \mathrm{mRNA}$ was no different from $\zeta W T$ mRNA (Figure $2 \mathrm{~B}$, left), the

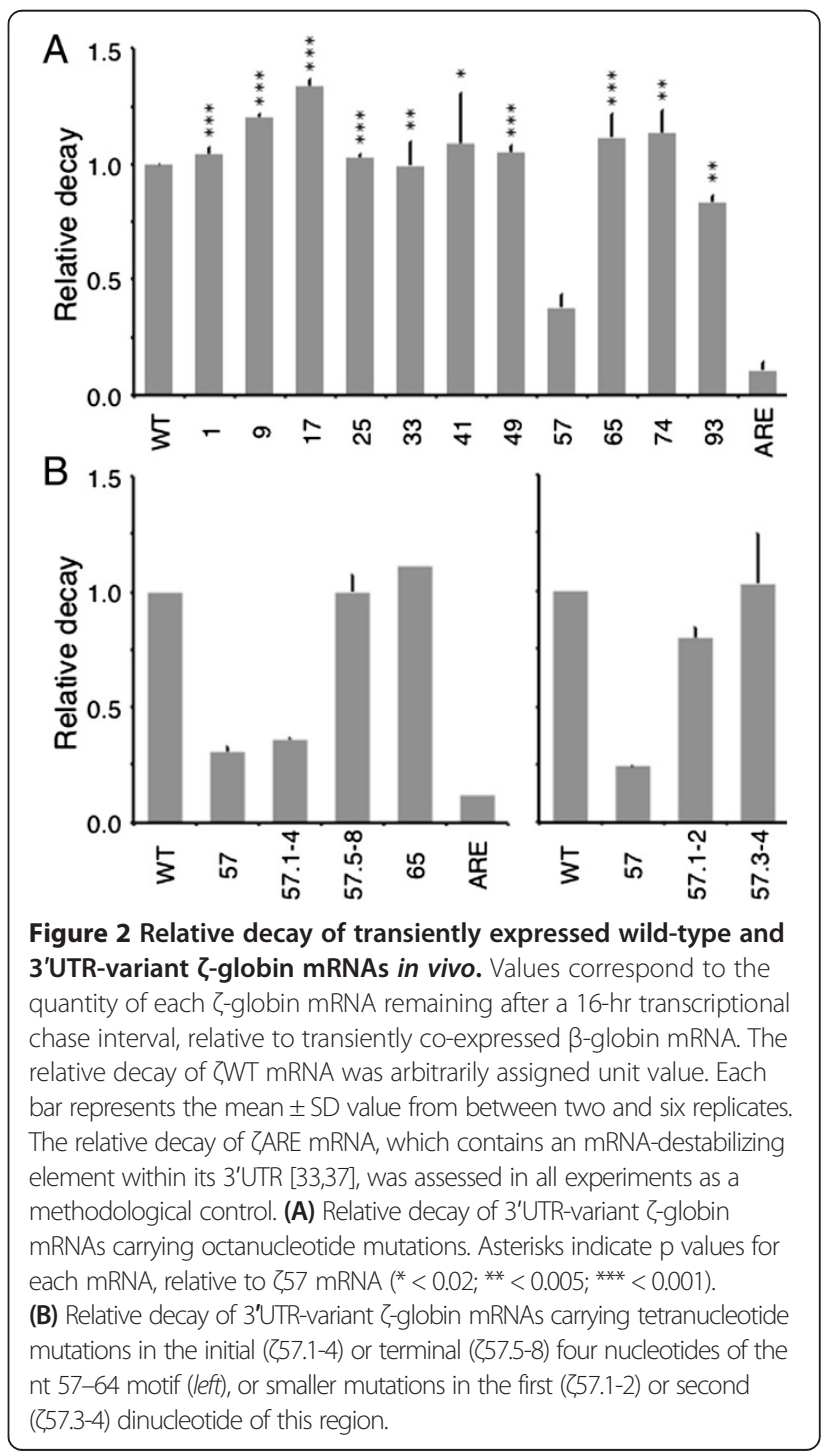


decay of $\zeta 57.1-4$ mRNA was significantly accelerated, and to a similar extent as that of parental $\zeta 57$ mRNA. Subsequent analyses demonstrated that smaller, dinucleotide substitutions to the nt 57-64 region did not affect mRNA decay, indicating that the ZMRE is functionally permissive for mutations of this magnitude (Figure 2B, right). Collectively, these screening data specify the importance of a site-specific tetranucleotide motif to the regulated accumulation of $\zeta$-globin mRNA.

\section{The ZMRE regulates the half-life of Z-globin mRNA}

Results from two-point screening analyses indicated the likelihood that the $\zeta 57$ determinant is essential for normal accumulation of $\zeta$-globin mRNA in cell cytoplasm. We validated this hypothesis by formally assessing $t_{1 / 2}$ values for wild-type and informative 3'UTR-variant $\zeta$-globin mRNAs in vivo. Analyses were conducted in triplicate in transiently transfected $\mathrm{HeLa}^{\text {tTA }}$ cells using a conventional pulse-chase strategy that assesses the levels of test mRNAs at defined intervals, relative to the level of endogenous tetindifferent $\beta$-actin mRNA [33]. Under these conditions, the $\zeta 57$ mRNA displayed a $t_{1 / 2}$ value that was approximately one-fourth that of $\zeta W T$ mRNA (3.7 $v 15.4 \mathrm{hr}$, respectively), while $\zeta 49$ and $\zeta 65$ mRNAs-containing ZMRE-flanking mutations-displayed relatively normal $t_{1 / 2}$ values of 11.6 and 10.2 hr (Figure 3). These data were affirmed by subsequent analyses demonstrating the deleterious effect the 57.1-4 mutation (but not the $557.5-8$ mutation) on the half-life of $\zeta$-globin mRNA (Figure 4). In these latter studies, the $t_{1 / 2}$ value of control $\zeta W T$ mRNA (11.2 hr) was reduced

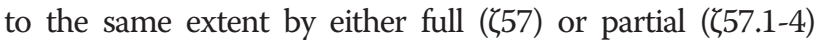
substitution of the nt 57-64 target region (3.6 and $3.7 \mathrm{hr}$, respectively), confirming the centrality of the CCCC tetranucleotide to ZMRE function. Additional pulse-chase studies-conducted in cells that stably express wild-type and

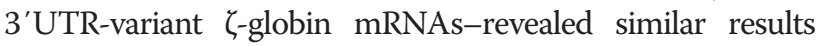
(not shown), providing a third measure of experimental validation. Collectively, the transcriptional chase analyses agree on the positioning of a post-transcriptional regulatory element within the $\zeta 57$ region, as well on its critical importance to the constitutive half-life of $\zeta$-globin mRNA.

\section{The mRNA-stabilizing activity of the ZMRE is not autonomous}

Previous analyses in transgenic mice demonstrated that the stability of human $\alpha$-globin mRNA was significantly reduced when its 3'UTR was exchanged for the corresponding region of $\zeta$-globin mRNA [18]. This effect was originally attributed to a generic difference in thenunspecified determinants of $\alpha$ - and $\zeta$-globin half-life [17] and, later, to sequence dissimilarities between homologous pyrimidine-rich regulatory elements within the $\alpha$ - and $\zeta$ -
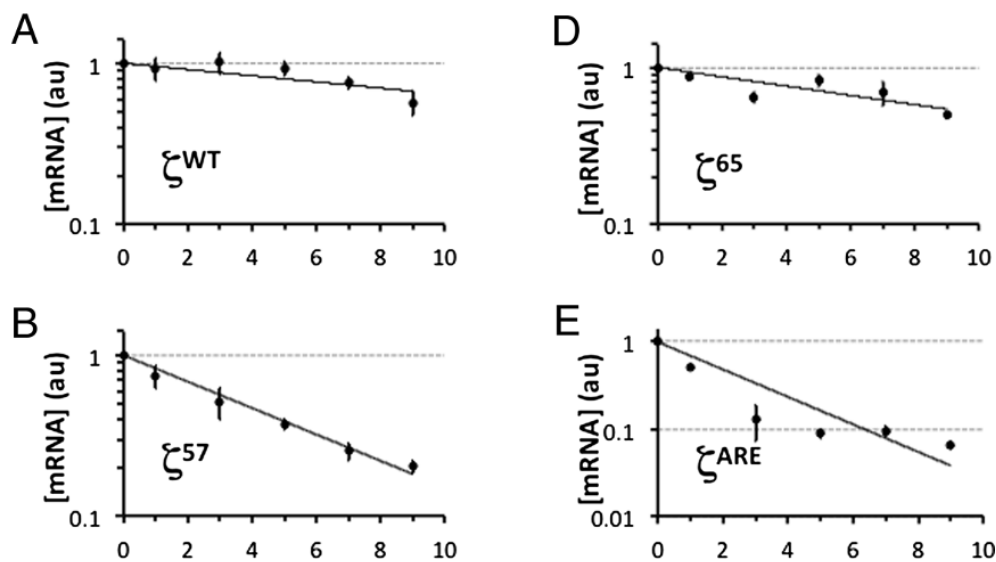

E
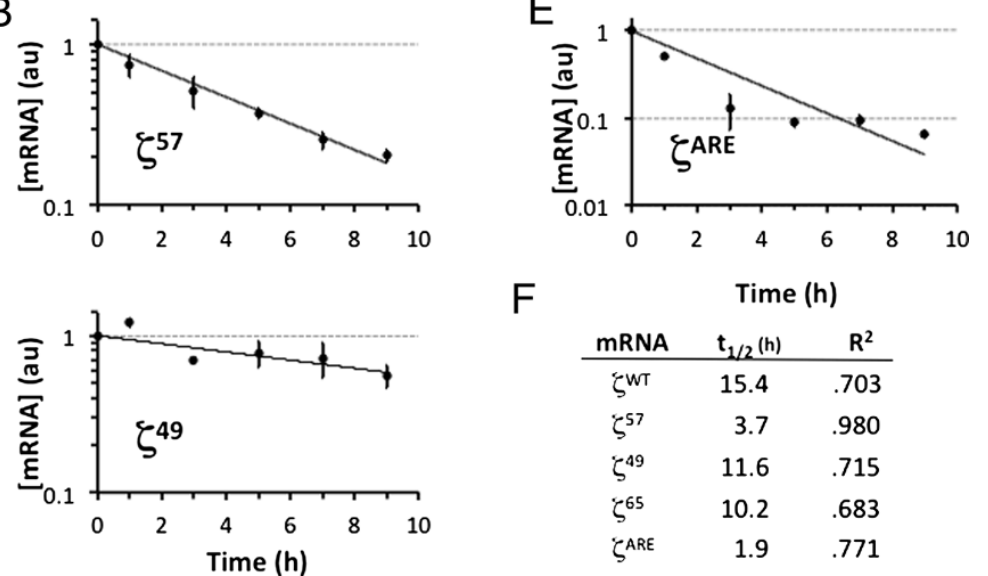

F

\begin{tabular}{crr} 
mRNA & \multicolumn{1}{c}{$\mathrm{t}_{1 / 2}(\mathrm{~h})$} & $\mathrm{R}^{2}$ \\
\hline$\zeta^{\mathrm{WT}}$ & 15.4 & .703 \\
$\zeta^{57}$ & 3.7 & .980 \\
$\zeta^{49}$ & 11.6 & .715 \\
$\zeta^{65}$ & 10.2 & .683 \\
$\zeta^{\text {ARE }}$ & 1.9 & .771
\end{tabular}

Figure 3 Half-life values for 3'UTR-variant $\zeta$-globin mRNAs. Transiently transfected HeLa ${ }^{\text {tTA }}$ cells were amended with tet, and levels of $\zeta$-globin mRNA assessed at defined intervals relative to levels of endogenous tet-indifferent $\beta$-actin mRNA. The normalized level of $\zeta-g l o b i n$ mRNA at each time point is plotted relative to the normalized level at $t=0$, which is arbitrarily assigned unit value. Each point depicts the mean \pm SD value for at least three replicates. (A) Stable control ZWT mRNA. (B) \57 mRNA. (C) $\zeta 49$ mRNA. (D) $\zeta 65$ mRNA. (E) Unstable control ZARE mRNA. (F) Half-life and R² values for curves in panels $A-E$, calculated from best-fit exponential decay curves with a fixed value of 1.0 at $t=0$. 


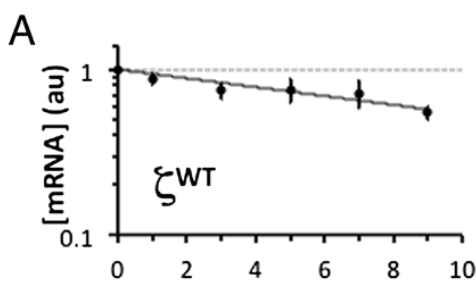

B
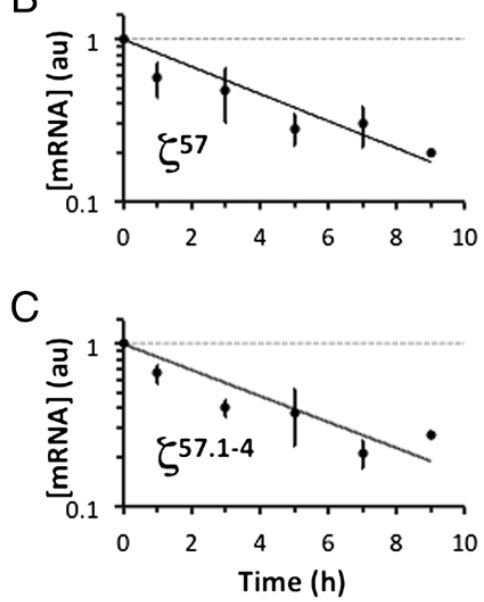

D

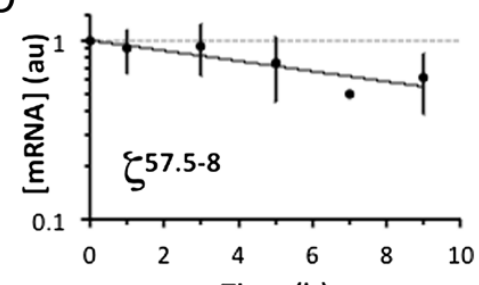

$\mathrm{E}$

Time (h)

\begin{tabular}{lrl} 
mRNA & $\mathrm{t}_{1 / 2}(\mathrm{~h})$ & $\mathrm{R}^{2}$ \\
\hline$\zeta^{W T}$ & 11.2 & .843 \\
$\zeta^{57}$ & 3.6 & .834 \\
$\zeta^{57.1-4}$ & 3.7 & .751 \\
$\zeta^{57.5-8}$ & 10.5 & .761
\end{tabular}

Half-life values for $\mathbf{5 7}$-derivative globin mRNAs. Stability analyses were conducted on informative $\zeta$-globin mRNAs as described in Figure 3. (A) Stable control $\zeta W T$ mRNA. (B) $\zeta 57$ mRNA. (C) $757.1-4$ mRNA. (D) $757.5-8$ mRNA. (E) Half-life and $R^{2}$ values for curves in panels A-D, calculated from best-fit exponential decay curves.

globin 3'UTRs [18]. We posited that this effect might alternately arise from a ZMRE function that was highly sensitive to its regional mRNA structural context; i.e., that the regulatory properties of the ZMRE might be reduced when repositioned near heterologous mRNA. To test this possibility, we assessed whether the half-life of a reporter coding-region mRNA contiguous with the $\zeta 57$ 3'UTR would be reduced relative to an identical mRNA flanked by a 弓WT 3'UTR (Figure 5A). A two-point decay analysis indicated that the stabilities of 3 'UTR-chimeric $\beta$-globin mRNAs were indifferent to the presence of stabilizing ( $\zeta W T$ ) or destabilizing ( $\zeta 57$ and $\zeta 57.1-4)$ 3'UTRs, validating an activity for the ZMRE that is dependent upon its RNA context (Figure $5 \mathrm{~B}$ ). While it is difficult to generalize this effect to coding regions from all mRNAs, our observations suggest that the activity of the ZMRE is defined both by its primary structure (Figures 3 and 4 ) as well as by undefined effects of neighboring mRNA and/or mRNA-bound factors (Figure 5).

\section{The ZMRE maps to a region of $\zeta$-globin 3'UTR that displays conformational flexibility}

The observation that the $\zeta$-globin mRNA is destabilized by mutations within the ZMRE, but not by similar mutations in neighboring cytosine-rich regions, suggests a local high-order structure that favors functional interactions between the ZMRE and one or more trans-acting effector factors. An unrefined $\mathrm{m}$-fold analysis of $\zeta$-globin 3'UTR structure predicts that the ZMRE is embedded in a highly stable RNA stem, and directly participates in

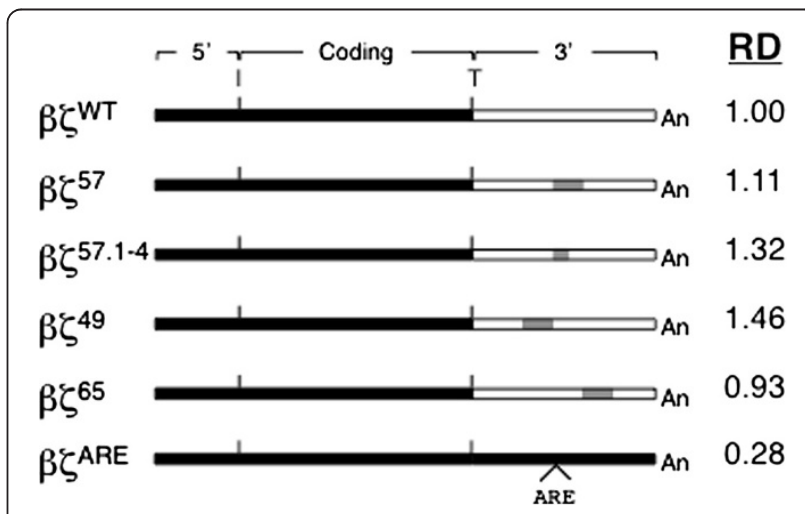

Figure 5 Relative decay of transiently expressed $\beta \zeta$-chimeric mRNAs in HeLa ${ }^{\text {tTA }}$ cells. (A) Structures of mRNAs encoded by chimeric $\beta \zeta$-globin genes; regions derived from $\beta$ - and $\zeta$-globin sequence are indicated in black and white, respectively. The positions of the translation initiation (I) and termination (T) sites, as well as the polyadenylate tail (An), are indicated. The $\zeta 57, \zeta 57.1-4, \zeta 49$, and $\zeta 65$ mutations are illustrated in gray. The $3^{\prime} U T R$ of the $\beta$ ZARE mRNA contains a defined 59-nt adenosine/uridine-rich element, inserted 15 nts $3^{\prime}$ to the translation stop codon; this motif destabilizes the full-length transcript, providing an unstable mRNA that is used as a methodological control [33]. $\mathrm{RD}=$ relative decay values obtained using the experimental strategy described in Figure 3. 
seven base-pair interactions (Figure 6A) [39,40]. We were skeptical that this arrangement would support sitespecific interactions with regulatory RNA-binding proteins, and consequently employed an enzymatic mapping technique to formally characterize the high-order RNA structure that encompasses the ZMRE [41]. Our analyses revealed regions of $\zeta$-globin 3'UTR that-by virtue of their sensitivities to single- and double-strand-specific RNases-were inconsistent with the unrefined structure (Figure 6B). A subsequent $\mathrm{m}$-fold analysis, constrained by unequivocal, experimentally determined regions of single- and double-stranded interactions, predicted an alternate structure with a favorable $\Delta G^{\circ}$ value $(-26.3 \mathrm{kcal} /$ $\mathrm{mol}$ ) in which the nt 57-64 target region is positioned on an asymmetrical interior loop, contiguous to a 16-nt stem (Figure 6C). This arrangement-which would be expected to facilitate conformational flexibility in the regions of RNA that flank the ZMRE [42]-would also account for our observation that transitional nucleotides (bordering the base-paired and unpaired regions) are assigned double-stranded interactions but display modest sensitivities to single-strand nucleases (e.g., C57 and C58). The resistance of the cytosine-rich ZMRE to double-strand-specific RNase V may additionally suggest potential hydrophobic 'stacking' interactions that can serve as a target for trans-acting regulatory factors [43]. The structural dynamics of this region, combined with experimental evidence favoring its assembly, indicates that the positioning of the ZMRE may be critical to its regulatory function.

\section{Cytoplasmic mRNA-binding proteins interact with the ZMRE}

The stabilities of many mRNAs-including those encoding $\alpha$ globin [27], $\beta$ globin [16], $\alpha(\mathrm{I})$ collagen [28], tyrosine hydroxylase [29], histone [30], and the transferrin receptor [31]-require the assembly of defined mRNP effector complexes on specific determinants within their 3'UTRs. To identify candidate trans-acting factors that might functionally interact with the ZMRE, we conducted affinity chromatography analyses on cell extracts from both non-erythroid HeLa and erythroid K562 cells, using three 32-nt single-stranded (ss) DNAs corresponding to comparable regions of $\zeta 57$, control $\zeta \mathrm{WT}$, and control $\zeta 653$ 'UTRs. Both the control $\zeta W T$ and $\zeta 65$ ssDNAs retained fewer than 10 distinct factors-two of which were not retained by the $\zeta 57$ ssDNA-suggesting sitespecific trans-acting interactions and potential regulatory functions (Figure 7A). Excised bands were subjected to nanoLC/MS/MS and provisionally identified as isoforms of AUF1 (hnRNP D), a protein that is ubiquitously expressed [44], displays known mRNA-binding characteristics [45,46], participates in post-transcriptional regulatory events that effect both mRNA stabilization [47] and destabilization [48], and has been implicated as a determinant of $\beta$ -

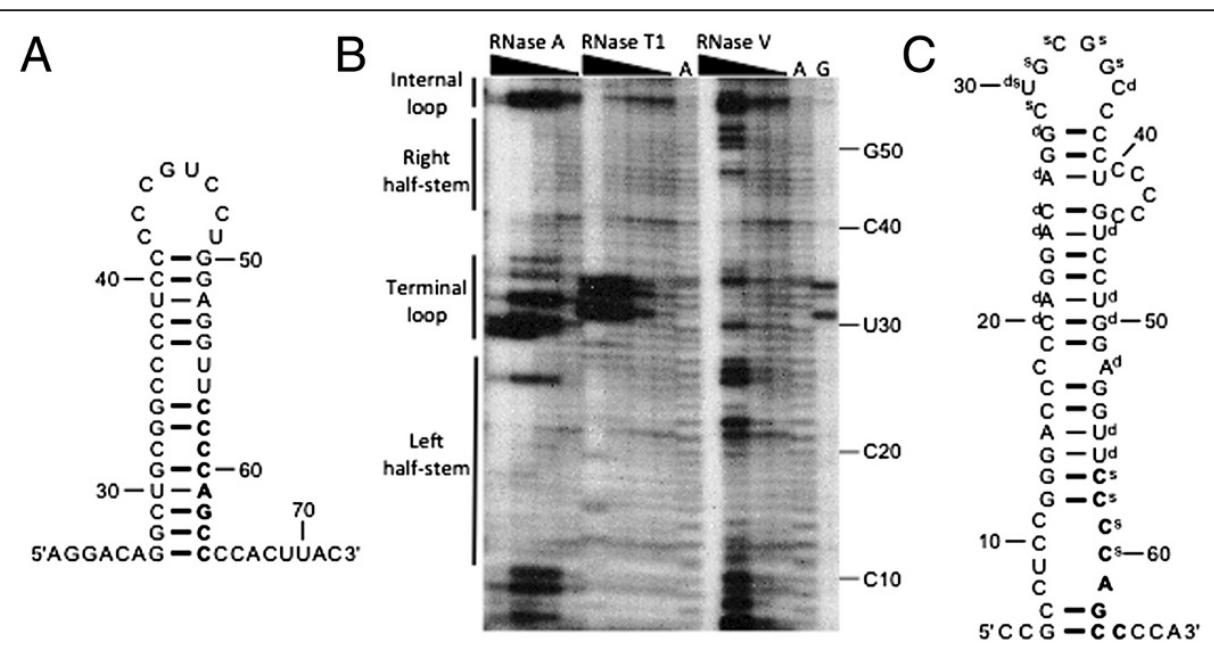

Figure 6 High-order structure of the $\zeta$-globin 3'UTR. (A) A thermodynamically favored structure resulting from unrefined m-fold analysis of the full-length $\zeta$-globin $3^{\prime} U T R$ (detail). The ZMRE is indicated in boldface. Double and triple hydrogen bond interactions are indicated by thin and thick connectors, respectively. (B) Enzymatic secondary-structure mapping of the $\zeta$-globin 3'UTR (detail). 5'-end [ ${ }^{32} \mathrm{P}$ ]-labeled RNAs corresponding to the Z-globin 3'UTR (and contiguous 18-nt polyadenylate tail) were digested with RNases A, T1, and V1 at four different concentrations (wedges), then resolved on an acrylamide-urea gel. Nucleotide assignments (right) were deduced by aligning the known $3^{\prime} U T R$ sequence to specific bands corresponding to guanine bases (lane G), which were generated by RNase T1 digestion of denatured $3^{\prime} U T R s$. An alkaline hydrolysis

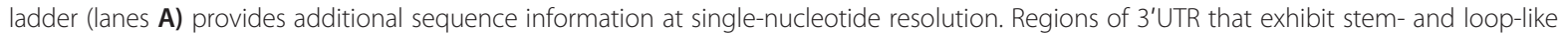
characteristics (i.e., sensitivities to double- and single-strand-specific nucleases, respectively) are indicated to the left. (C) Thermodynamically favored structure resulting from m-fold analysis of the full-length $\zeta$-globin 3'UTR, where base-pairing is enforced for nts 20-21, 24-26, and 38; and prohibited for nts 29-34 (detail). Nucleotides that displayed less-exacting single- or double-strand characteristics were not used for predictive purposes but are indicated by $s$ and $d$, respectively. 


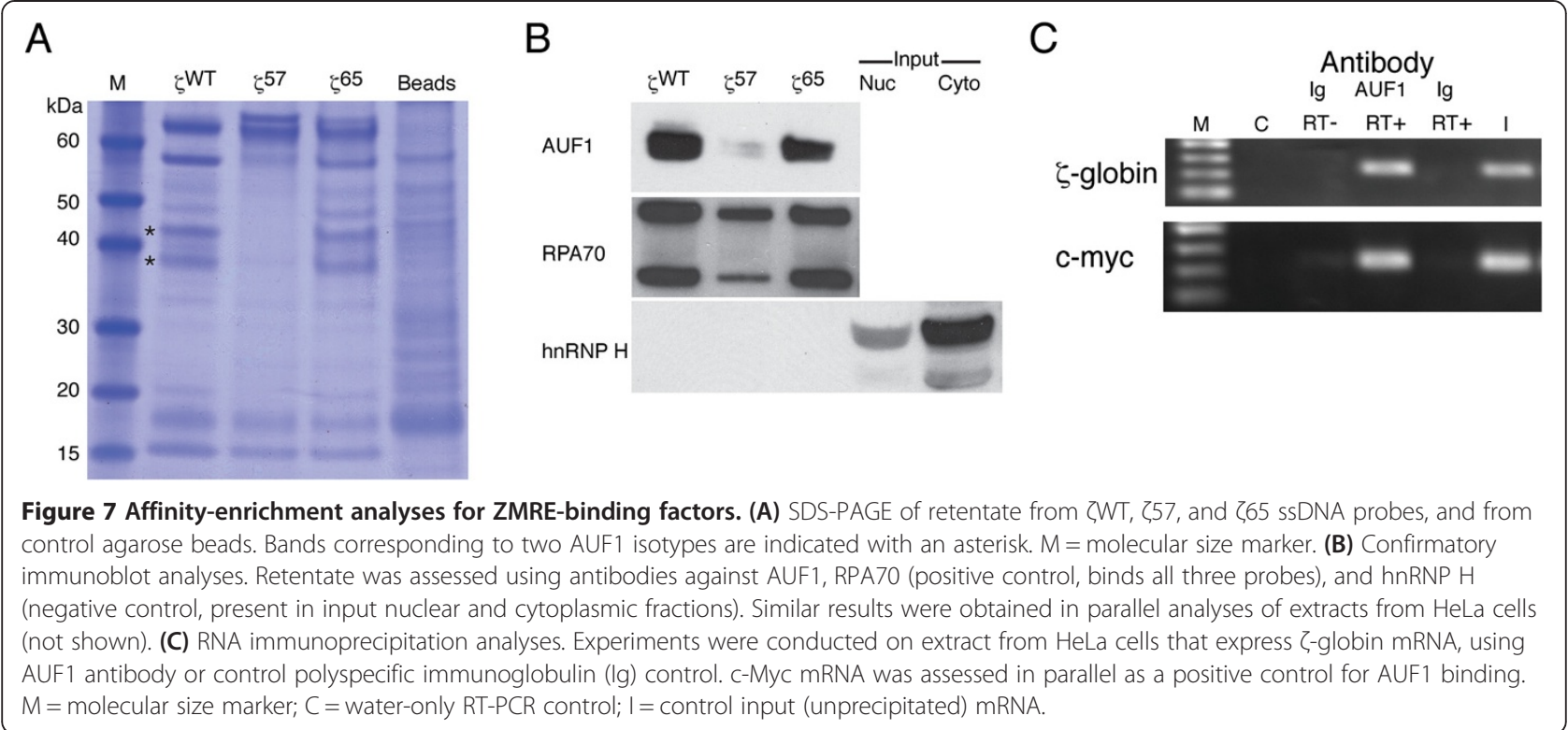

globin mRNA half-life [16]. The identity of AUF1-and its relative affinities for $\zeta \mathrm{WT}$, $\zeta 57$, and specificity-control $\zeta 65$ 3'UTRs-was subsequently validated by immunoblot analyses of the three retentates (Figure 7B). Additional analyses demonstrated that AUF1 immunoprecipitate of extract prepared from $\zeta$ WT-expressing $\mathrm{HeLa}^{\mathrm{tTA}}$ cells is enriched for both $\zeta W T$ and positive control c-myc mRNAs (Figure 7C), demonstrating the assembly of $\zeta$-globin/AUF1 mRNPs in vivo. These results document the interaction of AUF1 with the ZMRE and provide an attractive mechanistic link between the structure of the $\zeta$-globin $3^{\prime} \mathrm{UTR}$ and its mRNA-stabilizing functions. The importance of AUF1 to this process also suggests that the half-life of $\zeta$-globin mRNA may be regulated in definitive erythropoiesis through post-transcriptional programs that act on other mRNAs, including $\beta$-globin mRNA, that are central to normal red cell development.

\section{Discussion}

Recent reports that $\zeta$ globin acts as a physiological surrogate for deficient $\alpha$ globin [8,9] and efficiently inhibits the pathological polymerization of deoxyHb $\mathrm{S}[7,10]$ have engendered substantial interest in developing this embryonic globin as a unique and highly effective therapeutic for $\alpha$ thalassemia and sickle-cell disease, respectively. The premise that developmentally silenced globin genes can be derepressed in definitive erythroid cells is consistent with observations that $\zeta$ globin is expressed at high levels in several congenital and acquired conditions in humans [24-26] and can be reactivated by mutations that target transcriptional regulatory elements in animals [22-24]. Developmental stage-discordant embryonic globins can also be expressed from transgenes that have been modified to contain adult-stage promoters and enhancers $[6,17,22]$. While the specific mechanisms that underlie transcriptional repression of embryonic globin genes in definitive erythropoiesis remain a matter of active investigation, it is clear that this process can be reversed in adult erythroid progenitors.

An equally important determinant of $\zeta$-globin expre ssion-its post-transcriptional regulation-has been studied less extensively in both primitive and definitive erythropoiesis. Processes that impart high stability to globin mRNAs are particularly important in adult erythroblasts, permitting these transcripts to accumulate to high levels and to translate globin protein for 3-5 days following nuclear condensation and extrusion from orthochromatophilic erythroblasts [1]. As might be predicted, mutations that impair the high stabilities of globin mRNAs in transcriptionally silent cells will disproportionately impact the levels of their encoded proteins. For example, a naturally occurring antitermination mutation that shortens the $t_{1 / 2}$ of $\alpha$-globin mRNA to 25\% of its normal value ( $\alpha^{\text {Constant Spring }}$ ) coordinately reduces expression of the cognate globin monomer to $\sim 2 \%$ of the wild-type value $[49,50]$. Without some knowledge of the half-life of $\zeta$-globin mRNA, on the mechanism through which it is stabilized in definitive erythroid cells, it is difficult to predict whether transcriptional derepression of the $\zeta$ globin gene transcription will necessarily achieve the desired therapeutic effect.

We previously observed a modest difference in the stabilities of human $\alpha$-and $\zeta$-globin mRNAs that were compelled to express in definitive mouse erythroid cells [18]. This effect, which mapped to single-nt differences in homologous pyrimidine-rich elements (PREs) positioned within the two 3'UTRs, was attributed to a 6-fold reduction in the affinity of the $\zeta$-PRE for $\alpha \mathrm{CP}$ (hnRNP E), a cytoplasmic mRNA-binding protein that effects the high 
stability of $\alpha$-globin mRNA $[12,18]$. Importantly, $\zeta$-globin mRNA was not fully stabilized by exchange of the $\zeta$-PRE for the corresponding $\alpha$-globin determinant, suggesting the activities of other, structurally dissimilar mRNAstabilizing elements [18]. The present study validates this hypothesis, as it identifies a unique site-specific region of 3'UTR that is essential for the normal cytoplasmic accumulation of $\zeta$-globin (Figures 1 and 2). Subsequent half-life analyses, conducted in vivo in intact cultured cells, directly demonstrate the importance of the ZMRE (and, specifically, its 4-nt cytosine-rich core) to the cytoplasmic stability of $\zeta$-globin mRNA (Figures 3 and 4).

Our several measures suggest a $t_{1 / 2}$ value of $11-15 \mathrm{hr}$ for $\zeta$-globin mRNA in HeLa cells (Figures 3 and 4), which is surprisingly close to a $t_{1 / 2}$ value of $\sim 11 \mathrm{hr}$ for $\alpha$-globin mRNA obtained in MEL cells using a similar strategy [50]. The similar stabilities of $\alpha$ - and $\zeta$-globin mRNAs in these cultured cells-which do not express other globin mRNAs in significant quantities-contrasts sharply with their discordant stabilities in primary mouse cells that co-express high levels of endogenous $\alpha$ globin mRNA [18]. The cell context-dependent difference in the relative stabilities of the $\alpha$ - and $\zeta$-globin mRNAs suggests that they may be co-regulated through a shared post-transcriptional mechanism. We previously described a similar relationship among developmentally related $\beta$-like globin mRNAs, where the stabilities of transgenic embryonic $\varepsilon$ - and fetal $\gamma$-globin mRNAs are reduced in the presence of adult $\beta$-globin mRNA $[35,36]$. A similar relationship between the $\zeta$ - and $\alpha$-globin mRNAs would account for the significant reduction in the half-life of $\zeta$-globin mRNA when co-expressed with $\alpha$ globin mRNA of either human or mouse origin. The implications of this mechanism vis-à-vis $\alpha$ thalassemia are substantial, as expression of $\zeta$-globin mRNA from a therapeutic transgene would self-correct to higher or lower levels in patients with more or less severe deficits in $\alpha$ chain production, respectively.

Our results also indicate that the stability of $\zeta$-globin mRNA in definitive erythropoiesis reflects a balance between at least two separate post-transcriptional programs. The ZMRE that we identify is distinct from the previously identified $\zeta$-PRE in both its position within the 3'UTR as well as the specific trans-acting factors that it binds (Figure 7). Specifically, the ZMRE binds AUF1, a trans-acting mRNA-binding protein implicated in both the stabilization and destabilization of heterologous mRNAs in erythroid and non-erythroid cells $[16,47,48]$. This interaction may account for the observation that ZMRE-related activities are readily observed in non-erythroid HeLa cells (Figures 3 and 4), while $\alpha C P$ mediated programs that stabilize $\alpha$-globin mRNA are largely restricted to erythroid cells [11]. Importantly, AUF1 stabilizes $\beta$-globin mRNA through interaction with a 3'UTR determinant [16], suggesting potential mechanistic overlap between programs that direct the stabilities of $\alpha$-like and $\beta$-like mRNAs or, conceivably, the stabilities of embryonic and adult globin mRNAs. Both possibilities would be consistent with dynamic post-transcriptional competition between embryonic and adult globin transcripts [36] and would predict that the efficiency of $\zeta$-globin derepression in $\alpha$ thalassemia would be proportional to the severity of the $\alpha$-chain deficit.

One remarkable property of the ZMRE is its apparent non-autonomous function (Figure 5), suggesting 'allosteric' effects of surrounding structures on the activity of this determinant (Figure 6). While our studies do not investigate the positions of these function-modifying structures, we think it unlikely that they reside in coding region mRNA which is constantly remodeled by actively translating ribosomes. It seems reasonable to speculate that ZMRE activity is instead enforced by interactions with other regions of 3 'UTR (or factors that they bind), or by structural interface with the $\zeta$-globin 5 'UTR. The latter process might resemble the 'closed-loop' model for mRNA translation that invokes structural interactions between the 5'UTR, 3'UTR, and polyadenylate tail [51-55]. The experimentally determined structure surrounding the ZMRE is fully consistent with this possibility (Figure 6), predicting its positioning on an internal loop and/or within a hydrophobic nucleotide stack, where effector trans-acting factors might bind and subsequently alter the regional structure to reveal (or conceal) co-determinants of mRNA stability.

\section{Conclusion}

Collectively, the present studies address the relevance of post-transcriptional regulatory events to $\zeta$-globin expression in definitive erythroid cells, by defining structures and implicating mechanisms of $\zeta$-globin mRNA stability. Our functional analyses identify a previously unrecognized determinant of mRNA stability within the $\zeta$-globin 3'UTR whose position can be narrowed to a 4-nt site-specific cytosine sequence. Coordinate structural studies recognize that this region maps to a functionally germane mRNA form that is targeted by trans-acting regulatory factors that have been previously been implicated in stabilizing both globin and non-globin mRNAs. This information provides a foundation for subsequent mechanistic studies that will be critical for understanding the full utility of developmental stage-discordant $\zeta$-globin mRNA as a therapeutic for important disorders of globin gene expression.

\section{Methods}

\section{Gene construction}

Parental pTRE2A $\zeta$ was derived from pTRE2 (Clontech) by inserting the full-length human $\zeta$-globin gene (including $\sim 100$ nt of contiguous $5^{\prime}$ - and $3^{\prime}$-flanking regions) 
into the polylinker SacII-BamHI site. A $\sim 1.0 \mathrm{~kb}$ NcoISapI vector fragment was subsequently deleted to eliminate a default polyadenylation signal that competes with the native $\zeta$-globin poly(A) signal. 3'UTR-derivative $\zeta$ globin genes were constructed from pTRE2A $\zeta$ by exchanging the exon III BstEII-polylinker BamHI fragment with 330-bp synthetic DNAs (Genscript), each encompassing a mutated $\zeta$-globin 3'UTR. The construction of a corresponding full-length $\beta$-globin gene (pTRE2A $\beta$ ) is described elsewhere [15]. Genes encoding chimeric $\beta \zeta-$ globin mRNAs were generated from pTRE2A $\beta$ by exchanging the parental EcoRI-EcoNI DNA fragment (encompassing $\beta$-globin exon III and contiguous 3 '-flanking region) for a corresponding synthetic DNA containing the desired wild-type or derivative $\zeta$-globin 3'UTR. All recombinant DNAs were validated by automated sequencing.

\section{Cell culture}

$\mathrm{HeLa}^{\mathrm{tTA}}$ cells expressing the tetracycline transactivator fusion protein (tTA; Clontech) were maintained in DMEM supplemented with $10 \% \mathrm{FBS}$ and antibiotics, at $37^{\circ} \mathrm{C}$ in a humidified $5 \% \mathrm{CO}_{2}$ environment. Wild-type and 3'UTRderivative $\mathrm{pTRE} 2 \mathrm{~A} \zeta$ vectors used for stable transfections were modified by inserting a 1.5-kb DNA fragment encoding hygromycin (hyg) resistance into a unique vector XhoI site. Approximately $6 \times 10^{5}$ cells were transfected with $5.0 \mu \mathrm{g}$ of DNA using Superfect reagent under conditions recommended by the manufacturer (Qiagen), and selected with $400 \mu \mathrm{g} / \mathrm{mL}$ hyg. Hyg-resistant clones were tested by RT-qPCR for levels of $\zeta$-globin mRNA and control endogenous $\beta$-actin mRNA. Tetracycline (tet) response was assessed by quantifying the level of $\zeta$-globin mRNA, relative to the level of tet-indifferent $\beta$-actin mRNA, following a $48-\mathrm{hr}$ incubation in tet-supplemented media $(2 \mu \mathrm{g} / \mathrm{mL})$.

\section{mRNA decay analyses}

Two-point decay analyses were conducted on $5 \times 10^{5}$ preplated $\mathrm{HeLa}^{\mathrm{tTA}}$ cells maintained in tet-supplemented medium $(0.5 \mu \mathrm{g} / \mathrm{mL})$. Cells were transfected with $5.0 \mu \mathrm{g}$ DNA comprising equal quantities of wild-type (or 3' UTR-derivative) TRE2A $\zeta$ and control pTRE2A $\beta$ using Superfect reagent, and replated as two aliquots for overnight growth in tet-supplemented medium $(0.03 \mu \mathrm{g} / \mathrm{mL})$. PBS-washed aliquots were then incubated for five hr in tet-free medium; one aliquot was sacrificed immediately $(t=0)$ and a second aliquot after an additional 16-hr incubation in tet-supplemented medium $(2.0 \mu \mathrm{g} / \mathrm{mL})$. Conventional half-life analyses were conducted on $2 \times 10^{6}$ $\mathrm{HeLa}^{\text {tTA }}$ cells maintained in tet-supplemented medium $(0.5 \mu \mathrm{g} / \mathrm{mL})$, transfected with $10 \mu \mathrm{g}$ DNA using Superfect reagent, and then replated in aliquots in tet-supplemented medium $(0.03 \mu \mathrm{g} / \mathrm{mL})$ for overnight growth. PBS-washed aliquots were then incubated in tet-free medium for five $\mathrm{hr}$, supplemented with tet $(2.0 \mu \mathrm{g} / \mathrm{mL})$ and sacrificed at defined intervals. For both transient and stable analyses, cells were sacrificed by immersion in Trizol, and RNA prepared as recommended by the manufacturer (Invitrogen). Purified RNA was resuspended at $10 \mu \mathrm{g} / \mathrm{mL}$ in $\mathrm{H}_{2} \mathrm{O}$, and stored at $-80^{\circ} \mathrm{C}$.

\section{RT-qPCR}

Purified RNA (50 ng) was assayed using Taqman OneStep reagents on a model 7500 real-time PCR system, using protocols recommended by the manufacturer (Applied Biosystems; $\mathrm{AB}$ ). Analyses were conducted using assays for human $\zeta$ globin (AB catalogue HS00923579_m1), $\beta$ globin (HS00747223_g1), and $\beta$ actin (HS9999990 3_m1), and quantified by $\Delta \Delta \mathrm{Ct}$ methodology that we describe elsewhere [34]. Relative decay values were calculated as the quantity of each $\zeta$-globin mRNA remaining after a 16-hr transcriptional chase interval, relative to transiently co-expressed $\beta$-globin mRNA; the relative decay of $\zeta W T$ mRNA was arbitrarily assigned unit value. $\mathrm{p}$ values-where indicated-were calculated using standard Student t-test methods.

\section{Affinity enrichment}

Custom 5'-terminal biotinylated single-strand (ss) DNAs corresponding to $3^{\prime}$ UTRs from $\zeta^{\text {WT }}$ (5'TGGAGGTTCCC CAGCCCCACTTACCGCGTAAT3'), $\zeta^{57}$ (5'TGGAG GT TAGTGCACACCACTTACCGCGTAAT3'), and $\zeta^{65}\left(5^{\prime} \mathrm{T}\right.$ GGAGGTTCCCCAGCCAGTGCACACGCGTAAT3') mRNAs were commercially sourced (IDT). Approximately $1 \mu \mathrm{g}$ of each ssDNA was incubated overnight at $4^{\circ} \mathrm{C}$ in $500 \mu \mathrm{L}$ cytoplasmic extract supplemented with $50 \mu \mathrm{L}$ ImmunoPure Avidin Agarose beads (Pierce). The pelleted beads were washed $2 \times$ with PBS + Triton- $\times 100(0.05 \%)$ and $2 \times$ with PBS + Triton- $\times 100(1.0 \%)$, resuspended in $10 \mu \mathrm{L}$ loading buffer, and resolved on a precast $4-12 \%$ gradient SDS-polyacrylamide gel (Invitrogen). Cytoplasmic extracts were prepared from $\sim 1 \times 10^{7}$ cells lysed in buffer (1 mM Hepes pH 7.9, $0.15 \mathrm{mM} \mathrm{MgCl}, 1 \mathrm{mM} \mathrm{KCl}$ ) and clarified by centrifugation at $13000 \mathrm{xg}$ for five minutes at $4^{\circ} \mathrm{C}$; extract was stored in aliquots at $-80^{\circ} \mathrm{C}$.

\section{Proteomics}

Analyses were conducted by the University of Pennsylvania Proteomics Core Facility. Tryptic digests were studied by nanoLC/MS/MS using Thermo LTQ and Eksigebt nano LC-2 Da instruments, and data analyzed from the Uniprot_Sprot database using Sequest and Scaffold software packages. Statistical p-value cutoffs of $95.0 \%$ and $99.0 \%$ were applied for peptides and proteins, respectively.

\section{Western transfer}

Antibodies were used at the following dilutions: AUF1 (kind gift of G Dreyfuss; 1:2000), replication protein A (Santa Cruz SC-81373; 1:300), and hnRNP-H (Santa 
Cruz SC-10042; 1:500). Cytoplasmic extracts were resolved on precast $4-12 \%$ SDS-polyacrylamide gels, then transferred to nitrocellulose using an XCell II Module per manufacturer recommendations (Invitrogen). Membranes were blocked for 30 minutes at room temperature (RT) with Superblock T20 (Thermo Scientific), then supplemented with primary antibody for $60 \mathrm{~min}$ and with secondary HRP-conjugated antibody for an additional 30 minutes. Immunoblots were washed thrice for five minutes at RT with PBS + Tween-20 (0.1\%), and analyzed by ECL chemiluminescence (GE Healthcare).

\section{mRNA secondary structure}

A DNA template for in vitro transcription of the $\zeta^{\mathrm{WT}} 3^{\prime}$ UTR, containing an 18-nt polyadenylate tail, was directionally inserted into the XhoI-BglII polylinker site of pSP72. The BglII-linearized plasmid was transcribed in vitro as previously described [41], and the purified RNA then $5^{\prime}$-end labeled with $\left[\gamma^{-}{ }^{32} \mathrm{P}\right] \mathrm{ATP}$ using a Kinase Max kit (Ambion). The $\left[{ }^{32} \mathrm{P}\right]$-labeled RNA was digested with RNase A (100, 10, 1.0, and $0.1 \mathrm{ng} / \mathrm{mL})$, RNase T1 (100, 10, 1.0 , and $0.1 \mathrm{mU} / \mu \mathrm{L})$, or RNase $\mathrm{V} 1(10,1.0,0.1$, and $0.01 \mathrm{mU} / \mu \mathrm{L}$ ) as directed by the supplier (Ambion). RNases $\mathrm{A}$ and $\mathrm{T} 1$ exhibit cleavage specificities for pyrimidine and guanosine bases in single-stranded regions of RNA, respectively, while RNase V1 cleaves 3' to nucleotides within double-stranded regions of RNA. Reaction products were resolved on a $33 \mathrm{~cm} \times 40 \mathrm{~cm} \mathrm{6 \% :8} \mathrm{M} \mathrm{acryl-}$ amide:urea gel, in parallel with a migration-control 'ladder' generated by alkaline hydrolysis of $\left[{ }^{32} \mathrm{P}\right]$-labeled transcripts [41].

\section{Competing interests}

The authors declare that they have no competing interests.

\section{Authors' contributions}

Participated in research design: ZH, DS, SvZ, JER. Conducted experiments: ZH, DS, SvZ. Performed data analysis: ZH, JER. Wrote or contributed to the writing of the manuscript: JER. All authors read and approved the final manuscript.

\section{Acknowledgments}

The authors thank $O$ Abdulmalik for helpful discussions and critical reading of the manuscript, and $\mathrm{G}$ Dreyfuss for sharing antibody. This work was supported in part by NIH grants HL061399 (JER) and HL082754 (JER), and by a Research Scholar award from the Cooley's Anemia Foundation (SvZ). Mass spectrometries were conducted by the University of Pennsylvania Proteomics Core Facility under NIH grant P30CA016520.

Received: 20 February 2014 Accepted: 4 April 2014

Published: 21 April 2014

\section{References}

1. Russell JE, Liebhaber SA: Molecular genetics of thalassemia. In Advances in genome biology, Volume 2. Edited by Verma RS. Greenwich: JAI Press; 1993:283-353.

2. Bunn HF, Forget BG: Hemoglobin: molecular, genetic, and clinical aspects. Philadelphia: W. B. Saunders; 1986.

3. Leder A, Daugherty C, Whitney B, Leder P: Mouse zeta- and alpha-globin genes: embryonic survival, alpha thalassemia, and genetic background effects. Blood 1997, 90:1275-1282.
4. Felice AE, Cleek MP, Marino EM, McKie KM, McKie VC, Chang BK, Huisman $\mathrm{TH}$ : Different zeta-globin gene deletions among black Americans. Hum Genet 1986, 73:221-224.

5. Fei YJ, Kutlar F, Harris HF, Wilson MM, Milana A, Sciacca P, Schiliro G, Masala $B$, Manca L, Atlay C: A search for anomalies in the zeta, alpha, beta, and gamma globin gene arrangements in normal Black, Italian, Turkish, and Spanish newborns. Hemoglobin 1989, 13:45-65.

6. Russell JE, Liebhaber SA: Reversal of lethal alpha- and beta-thalassemias in mice by expression of human embryonic globins. Blood 1998, 92(9):3057-3063.

7. He Z, Russell JE: Anti-sickling effects of an endogenous human alpha-like globin. Nat Med 2004, 10:365-367.

8. He Z, Lian L, Asakura T, Russell JE: Functional effects of replacing human [alpha] and [beta] globins with their embryonic globin homologues in defined hemoglobin heterotetramers. Br J Haemat 2000, 109:882-890.

9. He Z, Russell JE: Expression, purification, and characterization of human hemoglobins Gower-1 ([zeta] $]_{2}[\text { epsilon }]_{2}$ ), Gower-2([alpha $]_{2}[\text { epsilon }]_{2}$ ), and Portland-2 ([zeta $]_{2}[\text { beta }]_{2}$ ) assembled in complex transgenic-knockout mice. Blood 2001, 97:1099-1105.

10. He Z, Russell JE: Effect of [zeta]-globin substitution on the $\mathrm{O}_{2}$-transport properties of $\mathrm{HbS}$ in vitro and in vivo. Biochem Biophys Res Comm 2004, 325:1376-1382.

11. Weiss IM, Liebhaber SA: Erythroid cell-specific determinants of alphaglobin mRNA stability. Mol Cell Biol 1994, 14:8123-8132.

12. Kiledjian M, Wang $X$, Liebhaber SA: Identification of two KH domain proteins in the alpha-globin mRNP stability complex. EMBO J 1995, 14:4357-4364.

13. Chkheidze AN, Lyakhov DL, Makeyev AV, Morales J, Kong J, Liebhaber SA: Assembly of the alpha-globin mRNA stability complex reflects binary interaction between the pyrimidine-rich 3'-untranslated region determinant and poly(C) binding protein [alpha] CP. Mol Cell Biol 1999, 19:4572-4581.

14. Yu J, Russell JE: Structural and functional analysis of an mRNP complex that mediates the high stability of human [beta]-globin mRNA. Mol Cell Biol 2001, 21:5879-5888.

15. Jiang $Y, X u X-S$, Russell JE: A nucleolin-binding 3'UTR element stabilizes [beta]-globin mRNA in vivo. Mol Cell Biol 2006, 26:2419-2429.

16. Van Zalen S, Jeschke GR, Hexner EO, Russell JE: AUF-1 and YB-1 are critical determinants of $\beta$-globin mRNA expression in erythroid cells. Blood 2012, 119:1045-1053.

17. Liebhaber SA, Wang Z, Cash F, Monks B, Russell JE: Developmental silencing of the embryonic zeta-globin gene: concerted action of the promoter and the 3 '-flanking region combined with stage-specific silencing of the transcribed region. Mol Cell Biol 1996, 16:2637-2646.

18. Russell JE, Morales J, Makeyev A, Liebhaber SA: Sequence divergence in the 3'UTRs of human zeta- and alpha-globin mRNA mediates a difference in their stabilities and contributes to efficient zeta-to-alpha gene developmental switching. Mol Cell Biol 1998, 18:2173-2183.

19. Yagi M, Gelinas R, Elder JT, Peretz M, Papayannopoulou T, Stamatoyannopoulos G, Groudine M: Chromatin structure and developmental expression of the human alpha-globin cluster. $\mathrm{Mol}$ Cell Biol 1986, 6:1108-1116.

20. Albitar M, Pechle C, Liebhaber SA: Theta-, zeta-, and epsilon-globin messenger RNAs are expressed in adults. Blood 1989, 74:629-637.

21. Lau ET, Kwok YK, Chui DH, Wong HS, Luo HY, Tang MH: Embryonic and fetal globins are expressed in adult erythroid progenitor cells and in erythroid cell cultures. Prenat Diagn 2001, 21:529-539.

22. Wang Z, Liebhaber SA: A 3'-flanking NF-kappaB site mediates developmental silencing of the human zeta-globin gene. EMBO J 1999, 18:2218-2228.

23. Liu J-J, Hou S-C, Shen C-KJ: Erythroid gene suppression by NF-kB. J Biol Chem 2003, 278:19534-19540.

24. Huang BL, Fan-Chiang IR, Wen SC, Koo HC, Kao WY, Gavva NR, Shen CK: Derepression of human embryonic zeta-globin promoter by a locuscontrol region sequence. Proc Natl Acad Sci U S A 1998, 95:14669-14674.

25. Chui DHK, Wong SC, Chung S-W, Patterson M, Bhargava S, Poon M-C: Embryonic zeta-globin chains in adults: a marker for alpha-thalassema-1 haplotype due to a >17.5 kb deletion. N Engl J Med 1986, 314:76-79.

26. Tang W, Cai SP, Eng B, Poon MC, Waye JS, Illum N, Chui DH: Expression of embryonic zeta-globin and epsilon-globin chains in a 10-year old girl with congenital anemia. Blood 1993, 81:1636-1640.

27. Wang $X$, Kiledjian M, Weiss IM, Liebhaber SA: Detection and characterization of a 3'-untranslated region ribonucleoprotein complex 
associated with human alpha-globin mRNA stability. Mol Cell Biol 1995, 15:1769-1777

28. Stefanovic B, Hellerbrand C, Holcik M, Briendl MA, Liebhaber S, Brenner DA Posttranscriptional regulation of collagen alpha1(I) mRNA in hepatic satellite cells. Mol Cell Biol 1997, 17:5201-5209.

29. Czyzyk-Krzeska MF, Beresh JE: Characterization of the hypoxia-inducible protein binding site within the pyrimidine-rich tract in the 3'-untranslated region of the tyrosine hydroxylase mRNA. J Biol Chem 1996, 271:3293-3299.

30. Pandey NB, Marzluff WF: The stem-loop structure at the $3^{\prime}$ end of histone mRNA is necessary and sufficient for regulation of histone mRNA stability. Mol Cell Biol 1987, 7(12):4557-4559.

31. Mullner EW, Neupert B, Kuhn LC: A specific mRNA binding factor regulates the iron-dependent stability of cytoplasmic transferrin receptor mRNA. Cell 1989, 58:373-382

32. Gossen $M$, Bujard H: Tight control of gene expression in mammalian cells by tetracycline responsive promoters. Proc Natl Acad Sci U S A 1992, 89:5547-5551.

33. Liu X, Russell JE: Cytoplasmic stabilities of 3'UTR-polymorphic prothrombin mRNAs. J Thromb Haemost 2010, 8:2580-2583.

34. Abdulmalik O, Lombardi AA, Russell JE: A reverse time-course method for transcriptional chase analyses of mRNA half-lives in cultured cells. PLoS One 2012, 7:e40827.

35. Russell JE: Post-transcriptional processes contribute to efficient developmental [gamma]-globin gene silencing in definitive erythroid cells. Eur J Haematol 2007, 79:516-525.

36. He Z, Russell JE: Dynamic post-transcriptional regulation of [epsilon]-globin gene expression in vivo. Blood 2007, 109:795-801.

37. Shaw $G$, Kamen $R$ : A conserved AU sequence from the 3 ' untranslated region of GM-CSF mRNA mediates selective mRNA degradation. Cell 1986, 46(5):659-667.

38. Holcik M, Liebhaber SA: Four highly stable eukaryotic mRNAs assemble 3' UTR RNA-protein complexes sharing cis- and trans-components. Proc Natl Acad Sci U S A 1997, 94:2410-2414.

39. Mathews DH, Sabina J, Zuker M, Turner DH: Expanded sequence dependence of thermodynamic parameters improves prediction of RNA secondary structure. J Mol Biol 1999, 288:911-940.

40. Zuker M: Mfold web server for nucleic acid folding and hybridization prediction. Nucleic Acids Res 2003, 31:3406-3415.

41. Liu X, Jiang $Y$, Russell JE: A potential regulatory role for mRNA secondary structures within the prothrombin 3'UTR. Thromb Res 2010, 126:130-136.

42. Zacharias M, Hagerman PJ: The influence of symmetrical internal loops on the flexibility of mRNA. J Mol Biol 1996, 257:276-289.

43. Kapoor D, Chandrayan SK, Ahmed S, Guptasarma P: Using DNA sequencing electrophoresis compression artifacts as reporters of stable mRNA structures affecting gene expression. Electrophoresis 2007, 28:3862-3867.

44. Lu JY, Schneider RJ: Tissue distribution of AU-rich mRNA-binding proteins involved in regulation of mRNA decay. J Bio/ Chem 2004, 279:12974-12979.

45. Wagner BJ, DeMaria CT, Sun Y, Wilson GM, Brewer G: Structure and genomic organization of the human AUF1 gene: alternative prre-mRNA splicing generates four protein isoform. Genomics 1998, 48:195-202.

46. Barreau C, Paillard L, Osborne HB: AU-rich elements and associated factors: are there unifying principles? Nucleic Acids Res 2006, 33:7138-7150

47. Xu N, Chen CY, Shyu AB: Versitile role for hnRNP D isoforms in the differential regulation of cytoplasmic mRNA turnover. Mol Cell Biol 2001 21:6960-6971.

48. Khabar KS: The AU-rich transcriptome: more than interferons and cytokines, and its role in disease. J Interferon Cytokine Res 2005, 25:1-10.

49. Liebhaber SA, Kan YW: Alpha thalassemia caused by an unstable alphaglobin mutant. J Clin Invest 1983, 71:461-466.

50. Kong J, Liebhaber SA: A cell type-restricted mRNA surveillance pathway triggered by ribosome extension into the $3^{\prime}$ untranslated region. Nat Struct Mol Biol 2007, 14:670-676.

51. Nagel RL, Sharma A, Kumar R, Fabry ME: Severe red cell abnormalities in transgenic mice expressing high levels of normal human delta chains. Blood 1995, 86:251a (abstract).

52. Zilka A, Garlapati S, Dahan E, Yaolsky V, Shapira M: Developmental regulation of heat shock protein 83 in Leishmania: $3^{\prime}$ processing and mRNA stability control transcript abundance, and translation is directed by a determinant in the 3 '-untranslated region. J Biol Chem 2001, 276:47922-47929.
53. Fritz $B$, Sheets $M$ : Regulation of the mRNAs encoding proteins of the BMP signaling pathway during the maternal stages of Xenopus oocyte development. Dev Biol 2001, 236:230-243.

54. Searfoss A, Dever T, Wickner R: Linking the $3^{\prime}$ poly $(A)$ tail to the subunit joining step of translation initiation: relations of Pab1p, eukaryotic translation initiation factor 5b (Fun12p), and Ski2p-Slh1p. Mol Cell Biol 2001, 21:4900-4908.

55. Wakiyama M, Honkura N, Miura K: Interference with interaction between eukaryotic translation initiation factor $4 \mathrm{G}$ and poly $(\mathrm{A})$ binding protein in Xenopus oocytes leads to inhibition of polyadenylated mRNA translation and oocyte maturation. J Biochem 2001, 130:737-740.

doi:10.1186/1756-8722-7-35

Cite this article as: He et al:: Structural determinants of human $\zeta$-globin mRNA stability. Journal of Hematology \& Oncology 2014 7:35.

\section{Submit your next manuscript to BioMed Central and take full advantage of:}

- Convenient online submission

- Thorough peer review

- No space constraints or color figure charges

- Immediate publication on acceptance

- Inclusion in PubMed, CAS, Scopus and Google Scholar

- Research which is freely available for redistribution
C Biomed Central 